\title{
EXPANSION OF ENTIRE FUNCTIONS OF SEVERAL COMPLEX VARIABLES HAVING FINITE GROWTH
}

\author{
BY \\ P. K. KAMTHAN AND MANJUL GUPTA(1)
}

\begin{abstract}
We consider the space $\chi$ of entire functions of two complex variables having a finite nonzero order point and type, equip it with the natural locally convex topology, such that $\chi$ becomes a Fréchet space. Apart from finding the characterization of continuous linear functionals, linear transformations on $\chi$, we have obtained the necessary and sufficient conditions for a double sequence in $\chi$ to be a proper base.
\end{abstract}

1. Introduction and terminology. Let $\chi$ denote the space of all entire functions of two variables $z_{1}, z_{2} \in \mathbf{C}$ (C is the ordinary complex plane equipped with its usual topology) endowed with the topology $\mathscr{F}$ of uniform convergence on compact sets in $\mathbf{C}^{2}$. The topology $\mathscr{F}$ can also be regarded as the topology generated by the family $\left\{M\left(f ; R_{1}, R_{2}\right): R_{1}, R_{2}>0\right\}$, where

$$
M\left(f ; r_{1}, r_{2}\right)=\sup _{\left|z_{1}\right| \leq r_{1}, z_{2} \mid \leq r_{2}}\left|f\left(z_{1}, z_{2}\right)\right|,
$$

and hence $\mathscr{F}$ can also be expressed in terms of arithmetical mean values of $f$; but certainly not in terms of geometric mean values of $f$ (for counterexample, see [5]). In an earlier paper [4] one of us has made a systematic study of the topological structure of the space $\chi$ leading to some characterizations of linear functionals, operators and proper bases in $\chi$. In all these results, functions of finite orders and types nowhere occupy a privileged position, although from the point of view of classical analysis, such functions are of immense importance. Our aim is, therefore, to consider the family of such class of entire functions of two variables (for the sake of simplicity, we consider the case of two variables only) and make a topological study thereon. Various notions of orders and types regarding functions in $\chi$ are available nowadays (see for instance the book by Fuks [2] and references given therein). We, however, follow those mentioned by Fred Gross [3]. Indeed, Fred Gross has given characterization of orders and types in terms of the coefficients of the expansion of an entire function $f \in \chi$, and this very well fits in our analysis in a most general setting.

For simplification, it is sometimes convenient to introduce various equivalent locally convex topologies on $\chi$ apart from $\mathscr{F}$ mentioned earlier. For instance, let

Received by the editors August 3, 1972 and, in revised form, February 27, 1973.

AMS (MOS) subject classifications (1970). Primary 32Al5; Secondary 46A05, 46A35.

Key words and phrases. Entire functions, order, type, mean values, locally convex topology, bases, linear functionals, transformations, proper bases.

(1) The research work of this author has been supported by the Junior Fellowship of CSIR, Government of India, and a junior research assistantship, I.I.T., Kanpur.

Copyright $\odot$ 1974, American Mathematical Society 
for each $f \in \chi$

$$
\|f\|=\sup \left\{\left|a_{00}\right|,\left|a_{m n}\right|^{1 /(m+n)}, m, n \geq 0, m+n \neq 0\right\} .
$$

For $R_{1}, R_{2}>0$, let

$$
p\left(f ; R_{1}, R_{2}\right)=\sum_{n=0}^{\infty} \sum_{m=0}^{\infty}\left|a_{m n}\right| R_{1}^{m} R_{2}^{n}
$$

where

$$
f\left(z_{1}, z_{2}\right)=\sum_{n=0}^{\infty} \sum_{m=0}^{\infty} a_{m n} z_{1}^{m} z_{2}^{n}, \quad a_{m n} \in \mathbf{C}
$$

with $\left|a_{m n}\right|^{1 /(m+n)} \rightarrow 0$ as $m+n \rightarrow \infty$. Then $\|f\|$ is a total paranorm and $p\left(f ; R_{1}, R_{2}\right)$ is a norm on $\chi$. Let $T_{1}$ and $T_{2}$ be the topologies generated by $\|f\|$ and the family $\left\{p\left(f ; R_{1}, R_{2}\right) ; R_{1}, R_{2}>0\right\}$ respectively. It is easy to see that $\mathscr{F} \simeq \boldsymbol{T}_{\mathbf{1}}$ $\simeq T_{2}$. Indeed, let $\left\{f_{p}\right\}$ be a net in $\chi$, such that $f_{p} \rightarrow f$ in $T_{1}$. Choose $\varepsilon>0, \eta>0$, $R_{1}, R_{2}>0$ arbitrarily, such that

$$
\eta R_{1}, \eta R_{2}<1, \quad \eta+\eta^{2} R_{1} R_{2} /\left\{\left(1-\eta R_{1}\right)\left(1-\eta R_{2}\right)\right\}<\varepsilon .
$$

Now for $p \geq Q=Q(\eta)$

$$
\left|a_{00}^{(p)}-a_{00}\right|<\eta, \quad\left|a_{m n}^{(p)}-a_{m n}\right|<\eta^{m+n} ; \quad m, n \geq 0, m+n \neq 0 .
$$

Then for $\left|z_{1}\right| \leq R_{1},\left|z_{2}\right| \leq R_{2}$,

$$
\left|f_{p}\left(z_{1}, z_{2}\right)-f\left(z_{1}, z_{2}\right)\right|<\eta+\frac{\eta R_{1}}{1-\eta R_{1}} \cdot \frac{\eta R_{2}}{1-\eta R_{2}}<\varepsilon, \quad \text { for all } p \geq Q .
$$

Hence $\mathscr{F} \subset T_{1}$. On the other hand, let $f_{p} \rightarrow f$ in $\mathscr{F}$. Then given $\varepsilon>0$, we may find $R_{1}, R_{2}>0$ with $R_{1}, R_{2}>1 / \varepsilon$ and a $Q=Q\left(\varepsilon, R_{1}, R_{2}\right)$, such that

$$
\begin{array}{cl}
\left|a_{00}^{(p)}-a_{00}\right|<\varepsilon, & \text { for } p \geq Q ; \\
\left|f_{p}\left(z_{1}, z_{2}\right)-f\left(z_{1}, z_{2}\right)\right| \leq 1, & \text { for } p \geq Q \text { and }\left|z_{i}\right| \leq R_{i}, i=1,2 .
\end{array}
$$

Using Cauchy's fundamental inequality for two variables [2, p. 49], namely, $\left|a_{m n}\right| R_{1}^{m} R_{2}^{n} \leq M\left(f ; R_{1}, R_{2}\right)$, one finds for $m+n \neq 0$,

$$
\left|a_{m n}^{(p)}-a_{m n}\right|^{1 /(m+n)} \leq R_{1}^{-m /(m+n)} R_{2}^{-n /(m+n)} \leq 1 / R \leq \varepsilon, \text { for } p \geq Q,
$$

where $R=\min \left(R_{1}, R_{2}\right) \geq 1 / \varepsilon$. Thus $f_{p} \rightarrow f$ in $T_{1}$. We have, therefore, shown that $\mathscr{F} \simeq T_{1}$. The other part, namely $\mathscr{F} \simeq T_{2}$, follows from the following inequalities:

$$
M\left(f ; r_{1}, r_{2}\right) \leq p\left(f ; r_{1}, r_{2}\right) \leq \frac{R_{1}}{R_{1}-r_{1}} \cdot \frac{R_{2}}{R_{2}-r_{2}} M\left(f ; R_{1}, R_{2}\right),
$$

where $R_{i}>r_{i}>0(i=1,2)$. Inequalities (1.4) follow from the Cauchy inequality referred to above. 
We now mention below the result of Fred Gross [3] on which our analysis depends. Let $\rho_{1}, \rho_{2} ; \sigma_{1}, \sigma_{2}$ be four positive finite numbers which are arbitrary but fixed throughout our discussion. Let $Y=Y\left(\rho_{1}, \rho_{2} ; \sigma_{1}, \sigma_{2}\right)$ be the subclass of $\chi$ consisting of those functions $f$ such that for each $\varepsilon>0$

$$
M\left(f ; r_{1}, r_{2}\right)<\exp \left\{\left(\sigma_{1}+\varepsilon\right) r_{1}^{\rho_{1}}+\left(\sigma_{2}+\varepsilon\right) r_{2}^{\rho_{2}}\right\},
$$

for all sufficiently large values of $r_{1}$ and $r_{2}$. Clearly $Y$ is a proper vector subspace of $\chi$. Since each $f \in Y$ is representable as given in (1.3), one has after Fred Gross the following:

$$
\left|a_{m n}\right|<\left[\frac{e \rho_{1}\left(\sigma_{1}+\varepsilon\right)}{m}\right]^{m / \rho_{1}}\left[\frac{e \rho_{2}\left(\sigma_{2}+\varepsilon\right)}{n}\right]^{n / \rho_{2}}
$$

valid for all sufficiently large $m+n$ and conversely if $f \in \chi$ and satisfies (1.6), then $f$ satisfies (1.5), i.e. $f \in Y$.

To consider the topological structure of $Y$, define for each $f \in Y$ and each $\delta>0$, the following term:

$$
\left\|f ; \sigma_{1}+\delta, \sigma_{2}+\delta\right\|
$$

$$
=\left|a_{00}\right|+\sum_{n=1} \sum_{m=1}\left|a_{m n}\right|\left[\frac{m}{\left(\sigma_{1}+\delta\right) e \rho_{1}}\right]^{m / \rho_{1}}\left[\frac{n}{\left(\sigma_{2}+\delta\right) e \rho_{2}}\right]^{n / \rho_{2}},
$$

where the double infinite series converges in view of (1.6). Then for each $\delta>0$, $\left\|f ; \sigma_{1}+\delta, \sigma_{2}+\delta\right\|$ defines a norm on $Y$. Let $\mathcal{G}$ be the topology on $Y$ generated by the family $\left\{\left\|f ; \sigma_{1}+\delta, \sigma_{2}+\delta\right\|: \delta>0\right\}$. We will throughout assume from now and onwards, unless otherwise stated, that $Y$ is equipped with the topology $\mathcal{G}$. It is then easily seen that $Y$ is a locally convex space. Moreover, it is metrizable, the metric being given by

$$
d(f, g)=\sum_{k=1}^{\infty} \frac{1}{2^{k}} \frac{\left\|f-g ; \sigma_{1}+1 / k, \sigma_{2}+1 / k\right\|}{1+\left\|f-g ; \sigma_{1}+1 / k, \sigma_{2}+1 / k\right\|} .
$$

Moreover, it can be easily verified that the topology induced on $Y$ by $\mathscr{F}$ is weaker than the original topology on $Y$ generated by $d$. In $\chi$, the sequence of partial sums of $f=\sum_{n=0}^{\infty} \sum_{m=0}^{\infty} a_{m n} \delta_{m n}, \delta_{m n}\left(z_{1}, z_{2}\right)=z_{1}^{m} z_{2}^{n}$, converges to $f$. This does not follow easily in the case of $Y$ when it is equipped with the topology 9 . We show that in fact it is true.

Theorem 1.1. Let $f=\sum_{n=0}^{\infty} \sum_{m=0}^{\infty} a_{m n} \delta_{m n} \in Y$. The sequence of partial sums of the series for $f$ converges to $f$ in $Y$ with respect to $G$.

Proof. If we prove that the assertion of the theorem is true in $Y(\delta)$ for every $\delta>0$, where $Y(\delta)$ the vector space $Y$ equipped with the norm (1.7), then the result will follow. Let $\delta>0$ be given. In view of (1.6), we can choose $\eta<\delta$ and $N=N(\eta)$ such that 


$$
\left|a_{m n}\right| \leq\left[\frac{e \rho_{1}\left(\sigma_{1}+\eta\right)}{m}\right]^{m / \rho_{1}}\left[\frac{e \rho_{2}\left(\sigma_{2}+\eta\right)}{n}\right]^{n / \rho_{2}}
$$

for all $m+n \geq N(\eta)$.

If $S_{N}$ is the partial sum of the series for $f, S_{N}=\Sigma \Sigma_{0<m+n<N^{a} m n} \delta_{m n}$, then

$$
\begin{aligned}
\left\|f-S_{N} ; \sigma_{1}+\delta, \sigma_{2}+\delta\right\| & =\sum_{i+j>N}\left|a_{i j}\right|\left[\frac{i}{e \rho_{1}\left(\sigma_{1}+\delta\right)}\right]^{i / \rho_{1}}\left[\frac{j}{e \rho_{2}\left(\sigma_{2}+\delta\right)}\right]^{j / \rho_{2}} \\
& \leq \sum_{i+j} \sum_{>N}\left[\frac{\sigma_{1}+\eta}{\sigma_{1}+\delta}\right]^{i / \rho_{1}}\left[\frac{\sigma_{2}+\eta}{\sigma_{2}+\delta}\right]^{j / \rho_{2}} \text { for } N \geq N(\eta) \\
& =\sum_{i+j} \sum_{>N} t_{1}^{i} t_{2}^{j} \rightarrow 0 \text { as } N \rightarrow \infty,
\end{aligned}
$$

where $t_{1}=\left(\left(\sigma_{1}+\eta\right) /\left(\sigma_{1}+\delta\right)\right)^{1 / \rho_{1}}<1$, and $t_{2}=\left[\left(\sigma_{2}+\eta\right) /\left(\sigma_{2}+\delta\right)\right]^{1 / \rho_{2}}<1$. This completes the proof.

2. Topological structure of $Y$ and its dual. First of all we have

Theorem 2.1. $Y$ equipped with the topology generated by the metric $d$ is a Fréchet space.

Proof. In view of the various considerations made in the preceding paragraph, it is sufficient to show that $Y$ is complete. It is a simple routine work. However, for the sake of completeness we may sketch the proof as follows. Let $\left\{f_{p}\right\}$, where

$$
f_{p}\left(z_{1}, z_{2}\right)=\sum_{n=0}^{\infty} \sum_{m=0}^{\infty} a_{m n}^{(p)} z_{1}^{m} z_{2}^{n}
$$

be a Cauchy sequence in $Y$ with respect to the metric $d$. Hence, it is a Cauchy sequence with respect to each $\left\|f ; \sigma_{1}+\delta, \sigma_{2}+\delta\right\|$ and it follows that for given $\delta>0, \varepsilon>0$, there exists $p_{0}=p_{0}(\varepsilon, \delta)$, such that

$$
\begin{gathered}
\left|a_{00}^{(p)}-a_{00}^{(q)}\right|+\sum_{m+n \geq 1} \sum_{m n}\left|a_{m n}^{(p)}-a_{m n}^{(q)}\right|\left[\frac{m}{\left(\sigma_{1}+\delta\right) e \rho_{1}}\right]^{m / \rho_{1}}\left[\frac{n}{\left(\sigma_{2}+\delta\right) e \rho_{2}}\right]^{n / \rho_{2}} \\
\leq \varepsilon \text { for all } p, q \geq p_{0} .
\end{gathered}
$$

Therefore, for each fixed $m$ and $n,\left\{a_{m n}^{(p)}\right\}$ is a Cauchy sequence in $\mathbf{C}$ and so $a_{m n}^{(p)} \rightarrow a_{m n}$ as $p \rightarrow \infty$ for $m, n \geq 0$.

Now for $p=p_{0}$,

$$
\left|a_{m n}\right| \leq\left|a_{m n}^{\left(p_{0}\right)}\right|+\left|a_{m n}^{\left(p_{0}\right)}-a_{m n}\right|<\left|a_{m n}^{\left(p_{0}\right)}\right|+\varepsilon
$$

and as $\left|a_{m n}^{\left(p_{0}\right)}\right|$ satisfies (1.6) so does then $\left|a_{m n}\right|$. Therefore the function $f$ given by (1.3) represents an element of $Y$ and it follows that $\left\|f_{p}-f ; \sigma_{1}+\delta, \sigma_{2}+\delta\right\| \rightarrow 0$ as $p \rightarrow \infty$. As $\delta$ is arbitrary, we are therefore done. 
Characterization of continuous linear functionals on $Y$. First, we prove the following theorem:

Theorem 2.2. A continuous linear functional $\phi$ on $Y(\delta)$ is of the form

$$
\phi(f)=\sum_{n=0}^{\infty} \sum_{m=0}^{\infty} c_{m n} a_{m n},
$$

where $f \in Y(\delta)$, if and only if

$$
\left|c_{m n}\right|\left[\frac{m}{e \rho_{1}\left(\sigma_{1}+\delta\right)}\right]^{-m / \rho_{1}}\left[\frac{n}{e \rho_{2}\left(\sigma_{2}+\delta\right)}\right]^{-n / \rho_{2}}
$$

is bounded for all $m, n \geq 0$.

Proof. Let $\phi$ be a continuous linear functional of the given form. Then it implies that there exists a constant $k>0$ such that

$$
|\phi(f)| \leq k\left\|f ; \sigma_{1}+\delta, \sigma_{2}+\delta\right\|
$$

for all $f \in Y(\delta)$. Choosing $f\left(z_{1}, z_{2}\right)=z_{1}^{m} z_{2}^{n}$, we find that

$$
\left|c_{m n}\right| \leq k\left\|\delta_{m n} ; \sigma_{1}+\delta, \sigma_{2}+\delta\right\|,
$$

for all $m, n \geq 0 \Rightarrow(2.2)$ holds.

Conversely, for each $f \in Y(\delta)$, let $\phi$ be defined as in (2.1) together with the condition (2.2). Then in view of (1.6) and (2.2) the infinite series in (2.1) converges and so $\phi$ is well defined. It can be easily shown that $\phi$ is a linear functional on $Y(\delta)$. Moreover, making use of (2.2) we find

$$
\begin{aligned}
|\phi(f)| & \leq \sum_{n=0}^{\infty} \sum_{m=0}^{\infty}\left|c_{m n}\right|\left|a_{m n}\right| \\
& \leq k\left\|f ; \sigma_{1}+\delta, \sigma_{2}+\delta\right\| .
\end{aligned}
$$

Hence $\phi$ is continuous on $Y(\delta)$. This completes the proof.

The main result of this section, which follows immediately from the above theorem, is stated as follows:

Theorem 2.3. Every continuous linear functional $\phi$ on $Y$ is of the form

$$
\phi(f)=\sum_{n=0}^{\infty} \sum_{m=0}^{\infty} c_{m n} a_{m n}
$$

where $f \in Y, f=\sum_{n=0}^{\infty} \sum_{m=0}^{\infty} a_{m n} \delta_{m n}$, and $\delta_{m n}\left(z_{1}, z_{2}\right)=z_{1}^{m} z_{2}^{n}$, if and only if

$$
\left|c_{m n}\right|<k\left[\frac{m}{e \rho_{1}\left(\sigma_{1}+\delta\right)}\right]^{m / \rho_{1}}\left[\frac{n}{e \rho_{2}\left(\sigma_{2}+\delta\right)}\right]^{n / \rho_{2}}
$$

holds for all $m, n \geq 0$, some constant $k>0$ and some $\delta>0$. 
3. Continuous linear transformations of $Y$ into itself. Let a continuous linear transformation from $Y\left(\delta_{1}\right)$ into $Y\left(\delta_{2}\right)$ be denoted by $T\left(\delta_{1}, \delta_{2}\right)$ and the family of all such transformations for a fixed pair $\delta_{1}$ and $\delta_{2}$ by $B\left(\delta_{1}, \delta_{2}\right)$. A continuous linear transformation from $Y$ into itself is denoted by $T$ and the family of such transformations is $B(Y)$. The main result of this section is stated as

Theorem 3.1. $A$ linear transformation $T \in B(Y)$ if and only if for each $\delta>0$ there exists a constant $k=k(\delta)$ and an $\varepsilon_{1}=\varepsilon_{1}(\delta)$ such that

$$
\left\|T\left(\delta_{m n}\right) ; \sigma_{1}+\delta, \sigma_{2}+\delta\right\|<k\left[\frac{m}{\left(\sigma_{1}+\varepsilon_{1}\right) e \rho_{1}}\right]^{m / \rho_{1}}\left[\frac{n}{\left(\sigma_{2}+\varepsilon_{1}\right) e \rho_{2}}\right]^{n / \rho_{2}}
$$

for all values of $m, n \geq 0$.

For proving this result, we shall need the following lemmas:

Lemma 3.1. If for $\alpha \in Y, d(\alpha)>k>0$, then the following must be true:

$$
\left\|\alpha ; \sigma_{1}+\delta, \sigma_{2}+\delta\right\|>k /(2-k)
$$

for some $\delta=\delta_{0}$, where $0<\delta_{0} \leq 1$, and therefore for all values of $\delta \leq \delta_{0}$.

Proof. Consider the sequence of norms $\left\|\alpha ; \sigma_{1}+1 / p, \sigma_{2}+1 / p\right\|$. This increases for increasing values of $p$. Choose $p_{0}$ such that

$$
\sum_{p_{0}+1}^{\infty} \frac{1}{2^{p}} \frac{\left\|\alpha ; \sigma_{1}+1 / p, \sigma_{2}+1 / p\right\|}{1+\left\|\alpha ; \sigma_{1}+1 / p, \sigma_{2}+1 / p\right\|}<\frac{k}{2}
$$

Then we have

$$
\begin{aligned}
d(\alpha) & <\frac{k}{2}+\frac{\left\|\alpha ; \sigma_{1}+1 / p_{0}, \sigma_{2}+1 / p_{0}\right\|}{1+\left\|\alpha ; \sigma_{1}+1 / p_{0}, \sigma_{2}+1 / p_{0}\right\|}\left\{\frac{1}{2}+\frac{1}{2^{2}}+\cdots+\frac{1}{2^{p_{0}}}\right\} \\
& \Rightarrow d(\alpha)<\frac{\left\|\alpha ; \sigma_{1}+1 / p_{0}, \sigma_{2}+1 / p_{0}\right\|}{1+\left\|\alpha ; \sigma_{1}+1 / p_{0}, \sigma_{2}+1 / p_{0}\right\|}+\frac{k}{2} .
\end{aligned}
$$

Since $d(\alpha)>k$, it implies $\left\|\alpha ; \sigma_{1}+1 / p_{0}, \sigma_{2}+1 / p_{0}\right\|>k /(2-k)$. With $\delta_{0}=1 / p_{0}$, the result follows.

Remark. From this lemma, it immediately follows that the convergence of a series in $Y(\delta)$ for each $\delta>0$ implies its convergence in $Y$. Combining this result with the fact that the topology on $Y$ is stronger than all the topologies on the $Y(\delta)$ 's, we can say that the convergence in $Y$ is equivalent to convergence in all of the $Y(\delta)$ 's.

Lemma 3.2. A linear transformation $T$ of $Y$ into itself is continuous if and only if to each $\delta_{2}>0$ there exists some $\delta_{1}>0$ such that $T \in B\left(\delta_{1}, \delta_{2}\right)$.

Proof. Since the topology on $Y$ is stronger than all the topologies on $Y(\delta)$ 's, it follows that any $T \in B(Y)$ is a continuous linear transformation from $Y$ into $Y\left(\delta_{2}\right)$ for each $\delta_{2}>0$. Choose $\delta_{2}$ arbitrarily and fix it. Therefore, for the 
necessary part we need to show the existence of some $\delta_{1}>0$ such that $T \in B\left(\delta_{1}, \delta_{2}\right)$. Let us assume that a linear transformation $T$ of $Y\left(\delta_{1}\right)$ into $Y\left(\delta_{2}\right)$ is not continuous for any $\delta_{1}>0$. Then by known properties of normed spaces $\left[1\right.$, p. 54], we can find a sequence $\{Y(1 / p)\}$ and $\alpha_{p}$ in $Y(1 / p)$ such that $\left\|\alpha_{p} ; \sigma_{1}+1 / p, \sigma_{2}+1 / p\right\| \rightarrow 0$ as $p \rightarrow \infty$, while $\left\|T\left(\alpha_{p}\right) ; \sigma_{1}+\delta_{2}, \sigma_{2}+\delta_{2}\right\| \geq 1$, for all sufficiently large $p$. This implies that $d\left(\alpha_{p}\right) \rightarrow 0$ as $p \rightarrow \infty$; but $\| T\left(\alpha_{p}\right) ; \sigma_{1}$ $+\delta_{2}, \sigma_{2}+\delta_{2} \| \geq 1 \Rightarrow T$ is not a continuous linear map from $Y$ into $Y\left(\delta_{2}\right)$. Since $\delta_{2}$ is arbitrary, it follows from the above arguments that for $T \in B(Y)$ and for any $\delta_{2}>0$, we should be able to find some $\delta_{1}>0$ such that $T \in B\left(\delta_{1}, \delta_{2}\right)$.

To prove the converse, suppose that a linear transformation $T$ of $Y$ into $Y$ is not continuous. Then there exists a sequence $\left\{\alpha_{p}\right\}$ of elements of $Y$ such that $d\left(\alpha_{p}\right) \rightarrow 0$ as $p \rightarrow \infty$ but $d\left(T\left(\alpha_{p}\right)\right) \geq k>0, p=1,2, \ldots$ By Lemma 3.1, we have

$$
\left\|T\left(\alpha_{p}\right) ; \sigma_{1}+\delta, \sigma_{2}+\delta\right\|>k /(2-k) \text { for all } \delta \leq \delta_{0}, 0 \leq \delta_{0} \leq 1 .
$$

This implies that $T$ is not a continuous linear map from $Y$ into $Y(\delta)$ for all $\delta \leq \delta_{0}$. From here, it can be easily shown that for $\delta$ 's $\leq \delta_{0}$, we cannot find any $\delta_{1}>0$ such that $T \in B\left(\delta_{1}, \delta\right)$. This completes the proof.

Proof of Theorem 3.1. Let $T \in B(Y)$ with $T\left(\delta_{m n}\right)=\alpha_{m n}$. Then, from Lemma 3.2, it follows that for every $\delta>0$, there exists $\delta_{1}=\delta_{1}(\delta)$ such that $T$ is a continuous linear map from $Y\left(\delta_{1}\right)$ to $Y(\delta)$.

There exists a constant $k=k(\delta)$ such that

$$
\begin{aligned}
& \left\|T\left(\delta_{m n}\right) ; \sigma_{1}+\delta, \sigma_{2}+\delta\right\| \leq k\left\|\delta_{m n} ; \sigma_{1}+\delta_{1}, \sigma_{2}+\delta_{1}\right\| \\
& \quad \Rightarrow\left\|\alpha_{m n} ; \sigma_{1}+\delta, \sigma_{2}+\delta\right\| \leq k\left[\frac{m}{\left(\sigma_{1}+\delta_{1}\right) e \rho_{1}}\right]^{m / \rho_{1}}\left[\frac{n}{\left(\sigma_{2}+\delta_{1}\right) e \rho_{2}}\right]^{n / \rho_{2}} .
\end{aligned}
$$

This proves the necessary part.

To prove the converse, let $\alpha \in Y$ be given by $\alpha=\sum_{n=0}^{\infty} \sum_{m=0}^{\infty} a_{m n} \delta_{m n}$. Then we have

$$
\left|a_{m n}\right|<\left[\frac{e \rho_{1}\left(\sigma_{1}+\varepsilon\right)}{m}\right]^{m / \rho_{1}}\left[\frac{e \rho_{2}\left(\sigma_{2}+\varepsilon\right)}{n}\right]^{n / \rho_{2}}
$$

for every $\varepsilon>0$ and for all sufficiently large $m+n$.

For fixed $\varepsilon>0$, there exists $N_{0}=N_{0}(\varepsilon)$ such that

$$
\left|a_{m n}\right|<\left[\frac{e \rho_{1}\left(\sigma_{1}+\varepsilon\right)}{m}\right]^{m / \rho_{1}}\left[\frac{e \rho_{2}\left(\sigma_{2}+\varepsilon\right)}{n}\right]^{n / \rho_{2}},
$$

for $(m+n) \geq N_{0}(\varepsilon)$. From (3.1) it follows that we can find $\varepsilon_{1}>\varepsilon$ such that

$$
\left\|\alpha_{m n} ; \sigma_{1}+\delta, \sigma_{2}+\delta\right\| \leq k\left[\frac{m}{\left(\sigma_{1}+\varepsilon_{1}\right) e \rho_{1}}\right]^{m / \rho_{1}}\left[\frac{n}{\left(\sigma_{2}+\varepsilon_{1}\right) e \rho_{2}}\right]^{n / \rho_{2}}
$$


for all $m, n \geq 0$. Combining (3.2) and (3.3) we get

$$
\begin{aligned}
\left|a_{m n}\right| \| \alpha_{m n} ; \sigma_{1} & +\delta, \sigma_{2}+\delta \| \leq k\left(\frac{\sigma_{1}+\varepsilon}{\sigma_{1}+\varepsilon_{1}}\right)^{m / \sigma_{1}}\left(\frac{\sigma_{2}+\varepsilon}{\sigma_{2}+\varepsilon_{1}}\right)^{n / \beta_{2}} \\
& \Rightarrow \sum_{n=0}^{\infty} \sum_{m=0}^{\infty}\left|a_{m n}\right|\left\|\alpha_{m n} ; \sigma_{1}+\delta, \sigma_{2}+\delta\right\| \text { converges for each } \delta>0 \\
& \Rightarrow \sum_{n=0}^{\infty} \sum_{m=0}^{\infty} a_{m n} \alpha_{m n} \text { converges to an element of } Y .
\end{aligned}
$$

Thus, we can define a map $T$ from $Y$ into itself as follows. For $\alpha \in Y$, define $T(\alpha)=\sum_{n=0}^{\infty} \sum_{m=0}^{\infty} a_{m n} \alpha_{m n}$. Then, obviously $T\left(\delta_{m n}\right)=\alpha_{m n}$ and $T$ is linear. Continuity of $T$ is immediate from the given condition (3.1) and the Lemma 3.2. This completes the proof.

4. Proper bases and their characterization. Let $\left\{\alpha_{m n}\right\}, m, n=0,1,2, \ldots$, be a sequence of entire functions in $Y$. The sequence $\left\{\alpha_{m n}\right\}$ is said to be linearly independent if $\sum_{n=0}^{\infty} \sum_{m=0}^{\infty} c_{m n} \alpha_{m n}=0 \Rightarrow c_{m n}=0$ for all sequences $\left\{c_{m m}\right\}$ of complex numbers for which the series $\sum_{n=0}^{\infty} \sum_{m=0}^{\infty} c_{m m} \alpha_{m m}$ converges in $Y$. A subspace $Y_{0}$ of $Y$ is said to be spanned by a sequence $\left\{a_{m n}\right\}$ if $Y_{0}$ consists of all linear combinations $\sum_{n=0}^{\infty} \sum_{m=0}^{\infty} c_{m m} \alpha_{m n}$ which are convergent in $Y$. A sequence $\left\{\alpha_{m n}\right\}$ which is linearly independent and spans a closed subspace $Y_{0}$ of $Y$ is called a basis in $Y_{0}$. Clearly $\left\{\delta_{m n}\right\}$ is a basis for $Y$. A proper basis in a subspace $X_{0}$ of $X$ is a basis $\left\{\alpha_{m n}\right\}$ in $X_{0}$, which possesses the following property: $\sum_{n=0}^{\infty} \sum_{m=0}^{\infty} c_{m n} \alpha_{m m}$ converges $\Leftrightarrow \sum_{n=0}^{\infty} \sum_{m=0}^{\infty} c_{m n} \delta_{m n}$ converges, for all sequences $\left\{c_{m n}\right\}$ of complex numbers. In our discussions which follow we shall make use of the fact that the convergence of $\sum_{n=0}^{\infty} \sum_{m=0}^{\infty} c_{m n} \delta_{m n}$ in $Y$ is equivalent to the condition:

$$
\left|c_{m n}\right|<\left[\frac{e \rho_{1}\left(\sigma_{1}+\varepsilon\right)}{m}\right]^{m / \rho_{1}}\left[\frac{e \rho_{2}\left(\sigma_{2}+\varepsilon\right)}{n}\right]^{m / \rho_{2}}
$$

valid for all sufficiently large $(m+n)$ and for every $\varepsilon>0$.

For the characterization of proper bases in terms of the growth conditions on $\left\{\alpha_{m n}\right\}$, we first prove the following two lemmas:

Lemma 4.1. For a sequence $\left\{\alpha_{m n}\right\} \subset Y$, the following three conditions are equivalent:

(A) For each $\delta>0$, there exists $k=k(\delta)$ and an $\varepsilon_{1}=\varepsilon_{1}(\delta)$ such that

$$
\left\|\alpha_{m n} ; \sigma_{1}+\delta, \sigma_{2}+\delta\right\|<k\left[\frac{m}{\left(\sigma_{1}+\varepsilon_{1}\right) e \rho_{1}}\right]^{m / \rho_{1}}\left[\frac{n}{e \rho_{2}\left(\sigma_{2}+\varepsilon_{1}\right)}\right]^{n / \rho_{2}},
$$

for all $m$ and $n(\geq 0)$. 
(B) For all sequences $\left\{c_{m n}\right\}$ of complex numbers, " $\sum_{n=0}^{\infty} \sum_{m=0}^{\infty} c_{m n} \delta_{m n}$ converges in $Y$ " implies " $\sum_{n=0}^{\infty} \sum_{m=0}^{\infty} c_{m n} \alpha_{m n}$ converges in $Y$ ".

(C) For all sequences $\left\{c_{m n}\right\}$ of complex numbers, " $\sum_{n=0}^{\infty} \sum_{m=0}^{\infty} c_{m n} \delta_{m n}$ converges in $Y$ " implies " $c_{m n} \alpha_{m n}$ tends to zero in $Y$ ".

Proof. From the sufficiency part of the proof of Theorem $3.1,(A) \Rightarrow$ (B) is obvious. $(B) \Rightarrow(C)$ is also quite obvious. So, in order to complete the proof, we need show that $(C) \Rightarrow(A)$. For this, suppose that $(C)$ is true, but $(A)$ is not true. Then the latter implies that for some $\delta$, say $\delta^{\prime}$, and every integer $k$ there exist sequences $\left\{m_{k}\right\}$ and $\left\{n_{k}\right\}$ of integers such that

$$
\left\|\alpha_{m_{k} n_{k}} ; \sigma_{1}+\delta^{\prime}, \sigma_{2}+\delta^{\prime}\right\| \geq k\left[\frac{m_{k}}{\left(\sigma_{1}+1 / k\right) e \rho_{1}}\right]^{m_{k} / \rho_{1}}\left[\frac{n_{k}}{\left(\sigma_{2}+1 / k\right) e \rho_{2}}\right]^{n_{k} / \rho_{2}} .
$$

Define a sequence $\left\{c_{m n}\right\}$ of scalars by

$$
\begin{aligned}
c_{m n} & =\frac{1}{\left\|\alpha_{m n} ; \sigma_{1}+\delta^{\prime}, \sigma_{2}+\delta^{\prime}\right\|} & & \text { when } m=m_{k} ; n=n_{k}, \quad k=1,2, \ldots, \\
& =0, & & \text { otherwise . }
\end{aligned}
$$

Then from (4.2), we have

$$
\begin{aligned}
&\left|c_{m_{k} n_{k}}\right| \leq \frac{1}{k}\left[\frac{\left(\sigma_{1}+1 / k\right) e \rho_{1}}{m_{k}}\right]^{m_{k} / \rho_{1}}\left[\frac{\left(\sigma_{2}+1 / k\right) e \rho_{2}}{n_{k}}\right]^{n_{k} / \rho_{2}} \\
&<\left[\frac{\left(\sigma_{1}+1 / k\right) e \rho_{1}}{m_{k}}\right]^{m_{k} / \rho_{1}}\left[\frac{\left(\sigma_{2}+1 / k\right) e \rho_{2}}{n_{k}}\right]^{n_{k} / \rho_{2}} \text { for } k>1 \\
& \Rightarrow\left|c_{m_{k} n_{k}}\right|<\left[\frac{\left(\sigma_{1}+\varepsilon\right) e \rho_{1}}{m_{k}}\right]^{m_{k} / \rho_{1}}\left[\frac{\left(\sigma_{2}+\varepsilon\right) e \rho_{2}}{n_{k}}\right]^{n_{k} / \rho_{2}} \\
& \text { for any } \varepsilon>0, \text { and for sufficiently large } k \\
& \Rightarrow\left|c_{m n}\right|<\left[\frac{\left(\sigma_{1}+\varepsilon\right) e \rho_{1}}{m}\right]^{m / \rho_{1}}\left[\frac{\left(\sigma_{2}+\varepsilon\right) e \rho_{2}}{n}\right]^{n / \rho_{2}},
\end{aligned}
$$

for any $\varepsilon>0$, and for sufficiently large $m$ and $n$.

Thus, $c_{m n}$ defined by (4.3) satisfies (4.1) and consequently $c_{m n} \alpha_{m n}$ should tend to zero in $Y$ by the hypothesis (C). But for $n=n_{k}$ and $m=m_{k}$,

$$
\left\|c_{m n} \alpha_{m n} ; \sigma_{1}+\delta^{\prime}, \sigma_{2}+\delta^{\prime}\right\|=\left|c_{m_{k} n_{k}}\right|\left\|\alpha_{m_{k} n_{k}} ; \sigma_{1}+\delta^{\prime}, \sigma_{2}+\delta^{\prime}\right\|=1
$$

which contradicts that $c_{m n} \alpha_{m n} \rightarrow 0$ in $Y$. This establishes that (C) $\Rightarrow(A)$.

Lemma 4.2. For a sequence $\left\{\alpha_{m n}\right\}$ in $Y$, the following conditions are equivalent:

( $\alpha$ ) For $\eta>0$ and all sufficiently small $\delta>0, \delta=\delta(\eta)$, 


$$
\left\|\alpha_{m n} ; \sigma_{1}+\delta, \sigma_{2}+\delta\right\| \geq\left[\frac{m}{\left(\sigma_{1}+\eta\right) e \rho_{1}}\right]^{m / \rho_{1}}\left[\frac{n}{\left(\sigma_{2}+\eta\right) e \rho_{2}}\right]^{n / \rho_{2}},
$$

where $m+n$ is sufficiently large and depends upon $\delta$.

$(\beta)$ For all sequences $\left\{c_{m n}\right\}$ of complex numbers, " $\sum_{n=0}^{\infty} \sum_{m=0}^{\infty} c_{m n} \alpha_{m n}$ converges in $Y " \Rightarrow \sum_{n=0}^{\infty} \sum_{m=0}^{\infty} c_{m n} \delta_{m n}$ converges in $Y "$.

$(\gamma)$ For all sequences $\left\{c_{m n}\right\}$ of complex numbers, " $c_{m n} \alpha_{m n}$ tends to zero in $Y$ " $\Rightarrow$ " $\sum_{n=0}^{\infty} \sum_{m=0}^{\infty} c_{m n} \delta_{m n}$ converges in $Y$ ".

Proof. $(\gamma) \Rightarrow(\beta)$ is obvious. We shall prove that $(\beta) \Rightarrow(\alpha)$ and $(\alpha) \Rightarrow(\gamma)$.

For proving $(\beta) \Rightarrow(\alpha)$, we assume that $(\beta)$ is true and $(\alpha)$ is not true. Then the latter implies that for some $\eta>0$, for arbitrary small $\delta_{0}$ and for arbitrarily large $m+n$, depending on $\delta_{0}$,

$$
\left\|\alpha_{m n} ; \sigma_{1}+\delta_{0}, \sigma_{2}+\delta_{0}\right\|<\left[\frac{m}{\left(\sigma_{1}+\eta\right) e \rho_{1}}\right]^{m / \rho_{1}}\left[\frac{n}{\left(\sigma_{2}+\eta\right) e \rho_{2}}\right]^{n / \rho_{2}} .
$$

Since $\left\|\alpha_{m n} ; \sigma_{1}+\delta, \sigma_{2}+\delta\right\|$ decreases as $\delta$ increases, it follows from here that

$$
\left\|\alpha_{m n} ; \sigma_{1}+\delta, \sigma_{2}+\delta\right\|<\left[\frac{m}{\left(\sigma_{1}+\eta\right) e \rho_{1}}\right]^{m / \rho_{1}}\left[\frac{n}{\left(\sigma_{2}+\eta\right) e \rho_{2}}\right]^{n / \rho_{2}}
$$

is true for each $\delta>0$ and arbitrarily large $m+n$ which depend on $\delta$. If $\eta$ is a fixed small positive number, we can find, corresponding to each number $k>0$, increasing sequences $\left\{m_{k}\right\}$ and $\left\{n_{k}\right\}$ such that

$$
\left\|\alpha_{m_{k} n_{k}} ; \sigma_{1}+\frac{1}{k}, \sigma_{2}+\frac{1}{k}\right\| \leq\left[\frac{m_{k}}{\left(\sigma_{1}+\eta\right) e \rho_{1}}\right]^{m_{k} / \rho_{1}}\left[\frac{n_{k}}{\left(\sigma_{2}+\eta\right) e \rho_{2}}\right]^{n_{k} / \rho_{2}} .
$$

Now choosing a positive number $\eta_{1}<\eta$, we define a sequence $\left\{c_{m n}\right\}$ of scalars as follows:

$$
\begin{aligned}
c_{m n} & =\left[\frac{\left(\sigma_{1}+\eta_{1}\right) e \rho_{1}}{m}\right]^{m / \rho_{1}}\left[\frac{\left(\sigma_{2}+\eta_{1}\right) e \rho_{2}}{n}\right]^{n / \rho_{2}}, & & \text { for } m=m_{k}, n=n_{k}, \\
& =0, & & \text { otherwise } .
\end{aligned}
$$

Then for any $\delta>0$,

$$
\sum_{n=0}^{\infty} \sum_{m=0}^{\infty}\left|c_{m n}\right|\left\|\alpha_{m n} ; \sigma_{1}+\delta, \sigma_{2}+\delta\right\|=\sum_{k \geq 1} \sum_{m_{k} n_{k}} \mid\left\|\alpha_{m_{k} n_{k}} ; \sigma_{1}+\delta ; \sigma_{2}+\delta\right\| .
$$

Fixing $\delta>0$, we omit from the above series those terms (finite in number) which correspond to those $m_{k}$ and $n_{k}$ for which $1 / k>\delta$. Then the remainder of the series is dominated by $\sum \sum_{k \geq 1}\left|c_{m_{k} n_{k}}\right|\left\|\alpha_{m_{k} n_{k}} ; \sigma_{1}+1 / k, \sigma_{2}+1 / k\right\|$. Now, making use of (4.6) and (4.7), and using the same arguments as in the proof of Theorem 1.1, the convergence of the above series can be easily verified. As $\delta>0$ is 
arbitrary, the series $\sum \sum_{m, n \geq 0} c_{m n} \alpha_{m n}$, when $\left\{c_{m n}\right\}$ is defined by (4.7), is convergent in $Y$ and consequently by our hypothesis $(\beta),\left\{c_{m n}\right\}$ should satisfy the condition (4.1). On the contrary, from the definition of the sequence $\left\{c_{m n}\right\}$, it is obvious that $\left\{c_{m n}\right\}$ does not satisfy the condition (4.1). Hence our assumption is wrong and $(\beta)$ $\Rightarrow(\alpha)$.

To prove $(\alpha) \Rightarrow(\gamma)$, we take a sequence $\left\{c_{m n}\right\}$ of scalars for which $c_{m n} \alpha_{m n} \rightarrow 0$ in $Y$ and the condition (4.1) does not hold. Then there exist sequences $\left\{m_{k}\right\}$ and $\left\{n_{k}\right\}$ of positive integers and a number $\lambda>0$ such that

$$
\left|c_{m_{k} n_{k}}\right| \geq\left[\frac{e \rho_{1}\left(\sigma_{1}+\lambda\right)}{m_{k}}\right]^{m_{k} / \rho_{1}}\left[\frac{e \rho_{2}\left(\sigma_{2}+\lambda\right)}{n_{k}}\right]^{n_{k} / \rho_{2}}
$$

Choose a positive number $\eta$ such that $\lambda>(3 \eta / 2)$. Then from $(\alpha)$, we can find a $\delta=\delta(\eta)$ such that

$$
\left\|\alpha_{m n} ; \sigma_{1}+\delta, \sigma_{2}+\delta\right\| \geq\left[\frac{m}{\left(\sigma_{1}+\eta\right) e \rho_{1}}\right]^{m / \rho_{1}}\left[\frac{n}{\left(\sigma_{2}+\eta\right) e \rho_{2}}\right]^{n / \rho_{2}},
$$

for sufficiently large $m+n$ which depends on $\delta$ and therefore on $\eta$. Therefore there exists a number $N(\eta)$ such that

$$
\begin{aligned}
\left\|\alpha_{m n} ; \sigma_{1}+\delta, \sigma_{2}+\delta\right\| \geq\left[\frac{m}{\left(\sigma_{1}+\eta\right) e \rho_{1}}\right]^{m / \rho_{1}}\left[\frac{n}{\left(\sigma_{2}+\eta\right) e \rho_{2}}\right]^{n / \rho_{2}}, & \text { for } m+n \geq N(\eta) .
\end{aligned}
$$

Now, consider

$$
\begin{aligned}
\max \left\|c_{m n} \alpha_{m n} ; \sigma_{1}+\delta, \sigma_{2}+\delta\right\| & =\max \left|c_{m n}\right|\left\|\alpha_{m n} ; \sigma_{1}+\delta, \sigma_{2}+\delta\right\| \\
& \geq \max \left|c_{m_{k} n_{k}}\right|\left\|\alpha_{m_{k} n_{k}} ; \sigma_{1}+\delta, \sigma_{2}+\delta\right\| \\
& \geq\left[\frac{e \rho_{1}\left(\sigma_{1}+\lambda\right)}{m_{k}}\right]^{m_{k} / \rho_{1}} \times\left[\frac{e \rho_{2}\left(\sigma_{2}+\lambda\right)}{n_{k}}\right]^{n_{k} / \rho_{2}} \\
& \times\left[\frac{m_{k}}{\left(\sigma_{1}+\eta\right) e \rho_{1}}\right]^{m_{k} / \rho_{1}} \times\left[\frac{n_{k}}{\left(\sigma_{2}+\eta\right) e \rho_{2}}\right]^{n_{k} / \rho_{2}} \\
& =\left(\frac{\sigma_{1}+\lambda}{\sigma_{1}+\eta}\right)^{m_{k} / \rho_{1}}\left(\frac{\sigma_{2}+\lambda}{\sigma_{2}+\eta}\right)^{n_{k} / \rho_{2}}>1 . \\
& >1 .
\end{aligned}
$$

This implies that $c_{m n} \alpha_{m n}$ does not tend to zero in $Y(\delta)$ corresponding to the above choice of $\delta$, and this contradicts our assumption. Hence $(\alpha) \Rightarrow(\gamma)$.

Combining the above two lemmas, we get a characterization of proper bases in the form of 
Theorem 4.1. $A$ base $\left\{\alpha_{m n}\right\}$ in a closed subspace $Y_{0}$ of $Y$ is proper if and only if $\left\{\alpha_{m n}\right\}$ satisfies the conditions (A) and $(\alpha)$.

5. Proper bases and linear homeomorphisms in $Y$. In this section, we characterize linear homeomorphisms in $Y$ in terms of proper bases as stated in the following theorem:

Theorem 5.1. If $T$ is linear homeomorphic mapping of $Y$ into itself, then $\left\{T\left(\delta_{m n}\right)\right\}$ is a proper base in some closed subspace $Y_{0}$ of $Y$. Conversely, if $\left\{\alpha_{m n}\right\}$ is a proper base in a closed subspace $Y_{0}$ of $Y$, then there exists a linear homeomorphic mapping $T$ of $Y$ onto $Y_{0}$ such that $T\left(\delta_{m n}\right)=\alpha_{m n}, m, n=0,1,2, \ldots$.

The proof of this result depends upon the condition (A) of the preceding section and runs on lines similar to those given in [6, Theorem 2.1]. It is therefore omitted. Through suitable combination of mappings, we also get the following interrelationship between proper bases and automorphisms (linear homeomorphic mappings of $Y$ onto itself).

Theorem 5.1'. If $\left\{\alpha_{m n}^{1}\right\}$ and $\left\{\alpha_{m n}^{2}\right\}$ are proper bases in $Y$, then there exists an automorphism $T$ of $Y$ such that $T\left(\alpha_{m n}^{1}\right)=\alpha_{m n}^{2}, m, n=0,1,2, \ldots$, and conversely if $T$ is an automorphism of $Y$ and $\left\{\alpha_{m n}^{1}\right\}$ is a proper base in $Y$, then $\left\{\alpha_{m n}^{2}\right\}$, $m, n=0,1,2, \ldots$, is also a proper base in $Y$, where $\alpha_{m n}^{2}=T\left(\alpha_{m n}^{1}\right)$.

Acknowledgment. We are grateful to the referee for making useful suggestions for the improvement of this paper.

\section{REFERENCES}

1. S. Banach, Théorie des opérations linéaires, Monografie Mat., PWN, Warsaw, 1932.

2. B. A. Fuks, Introduction to the theory of analytic functions of several complex variables, Fizmatgiz, Moscow, 1962; English transl., Transl. Math. Monographs, vol. 8, Amer. Math. Soc., Providence, R.I., 1963. MR 27 \#4945; 29 \#6049.

3. Fred Gross, Generalized Taylor series and orders and types of entire functions of several complex variables, Trans. Amer. Math. Soc. 120 (1965), 124-144. MR 32 \#2610.

4. P. K. Kamthan, A study on the space of entire functions of several complex variables, Yokohama Math. J. 21 (1973).

5.—, A note on the geometric means of entire functions of several complex variables, Trans. Amer. Math. Soc. 169 (1972), 503-508.

6. P. K. Kamthan and Manjul Gupta, Linear homeomorphisms in the space of analytic functions in bicylinders, Riv. Mat. Univ. Parma (to appear).

Department of Mathematics, Indian Institute of Technology, KanPUR 16, INDia 